Интернет-журнал «Транспортные сооружения» https://t-s.today

Russian journal of transport engineering

2020, №1, Tом 7 / 2020, No 1, Vol 7 https://t-s.today/issue-1-2020.html

URL статьи: https://t-s.today/PDF/05SATS120.pdf

DOI: 10.15862/05SATS120 (http://dx.doi.org/10.15862/05SATS120)

Ссылка для цитирования этой статьи:

Шестериков Ю.А., Стифеева О.А. Информационные системы и технологии обследования зданий и сооружений // Интернет-журнал «Транспортные сооружения», 2020 №1, https://t-s.today/PDF/05SATS120.pdf (доступ свободный). Загл. с экрана. Яз. рус., англ. DOI: 10.15862/05SATS120

For citation:

Shesterikov Yu.A., Stifeeva O.A. (2020). Information systems and technologies for inspection of buildings and structures. Russian journal of transport engineering, [online] 1(7). Available at: https://t-s.today/PDF/05SATS120.pdf (in Russian). DOI: 10.15862/05SATS120

\title{
УДК 72
}

Шестериков Юрий Алексеевич

ФГБОУ ВО «Национальный исследовательский Московский государственный строительный университет»

Москва, Россия

Научно-образовательный центр испытания сооружений Преподаватель

E-mail: norb-89@mail.ru

\section{Стифеева Ольга Алексеевна}

ФГБОУ ВО «Национальный исследовательский Московский государственный строительный университет»

Москва, Россия

Кафедра «Информационных систем, технологий и автоматизации» Преподаватель

E-mail: norb-89@mail.ru

\section{Информационные системы и технологии обследования зданий и сооружений}

Аннотация. В статье рассмотрены особенности применения информационных систем и технологий обследования зданий и сооружений. Автор отмечает, что в качестве основного критерия принятия решений о необходимости проведения капитального ремонта или о признании здания аварийным выступает категория технического состояния здания. Чтобы оценить категорию технического состояния конструкций, проводя обследование реальных строительных объектов, нужно учесть ряд всевозможных причин, которые приводят к изменению категории технического состояния. Сбор неформальной информации в базу знаний является стратегически важной и наиболее сложной задачей в разработке ЭС. Источниками знаний можно определить обширную базу практических и теоретических исследований в рамках технической диагностики конструкций зданий и сооружений, нормативную документацию, комплекс эвристических знаний и рассуждений специалистов.

Возможность автоматизировать решение задач экспертной деятельности в рамках диагностики строительных конструкций в условиях неопределённости необходимо искать в области систем искусственного интеллекта. На принимаемые решения оказывает влияние объективность и достоверность информации, предоставляемой экспертами, но достаточно часто такая информация находится под влиянием их субъективного мнения. Применение информационных технологий позволяет учитывать разброс индивидуальных мнений. Среди преимуществ использования информационных технологий при обследовании зданий можно 
назвать то, что исполнитель работ в «полевых» условиях на объекте может отследить сбор данных; возрастает продуктивность работы за счёт того, что значительно сокращается время для сбора и последующей обработки информации; возрастает точность контроля ряда параметров (геометрических, конструктивных) и появляется возможность автоматизации процесса получения информации об объекте, а также расчета и оценки прочностных характеристик исследуемых строительных конструкций.

Данные технологии позволяют дать строгое математическое описание расплывчатых утверждений касательно технического состояния здания на текущий момент.

Ключевые слова: строительные конструкции; экспертные системы; информационные технологии; обследование; параметры; аварийное состояние; капитальный ремонт; эксперт

Повышение конструкционной безопасности выступает приоритетным направлением обеспечения процесса комплексной безопасности зданий и сооружений. Важная предпосылка к этому - проведение обследования и технической диагностики строительных объектов. Категорию технического состояния (КТС) считают главным критерием в области принятия решения о необходимости проведения мероприятий в рамках приведения обследуемого строительного объекта к дальнейшей безопасной эксплуатации [2]. На данный критерий оказывает влияние большое число взаимосвязанных параметров, а также причин повреждённости строительных конструкций. Оценки таких факторов, формируемые экспертом, часто могут обладать некоторой погрешностью, противоречивостью и неопределённостью. Так, в процессе измерения количественных параметров почти всегда имеется погрешность, которая зависит от таких параметров, как применяемая приборная база и квалификация специалиста [3]. Неопределённость может иметь место вносить как из-за недостаточности сформулированных критериев оценки в нормативных документах, включающих заключение о включении строительной конструкции в ту или иную категорию технического состояния, так и из-за короткой шкалы ГОСТ, позволяющей отнести здание к нормативным, работоспособным, ограниченно работоспособным или аварийным [1].

Известно, что строительная конструкция может переходить из одного технического состояния в другое фактически происходит не «скачкообразно», а обретая достаточно большое количество промежуточных состояний, границы между которыми являются размытыми. По этой причине эксперт при назначении КТС конструкций должен принимать волевые решения, на основании которых увеличивается нежелательная доля субъективности в техническом заключении [12].

Эксперту, проводящему обследованию, необходимо установить ряд наиболее значимых признаков из выделенного набора параметров, характеризующих состояние здание, а для этого нужен опыт, способствующий развитию у специалиста личных или эвристических знаний. За счет таких знаний эксперт способен к выдвижению разумных предположений, может находить подходы к задачам и принимать эффективные решения даже в ситуации нечётких или неполных данных. Соответственно, целесообразно усилить и расширить профессиональные возможности специалистов, которые проводят обследование строительных объектов, применяя интеллектуальные технологии. Термин «интеллектуальные» подразумевает способность системы к поиску явных следствий из неявно представленных знаний с имитацией рассуждений человека [5].

Автоматизированный поиск экспертного решения может помощь в работе не только начинающему, но и опытному эксперту.

Возможность автоматизировать решение задач экспертной деятельности в рамках диагностики строительных конструкций в условиях неопределённости необходимо искать в 
области систем искусственного интеллекта. Термин «искусственный интеллект» или «разумные решения и логические рассуждения» ввел американский ученый Джон Маккарти в 1956 году [4].

В начале 80-х голов XX века в исследованиях по искусственному интеллекту было организовано самостоятельное направление, которое получило название «экспертные системы» (ЭС). Данные системы при решении сложных задач в области принятия решений в условиях многофакторности и неоднозначности способны получить результаты, качество и эффективность которых сходны с теми, которые получает опытный человек-эксперт. Этим решениям свойственна «прозрачность», то есть их можно объяснить пользователям на качественном уровне.

Экспертные системы имеют способность к пополнению своих знаний в процессе взаимодействия с экспертом. Их цель - не заменить эксперта в его непосредственной предметной деятельности, а расширить и усилить профессиональные возможности пользователей для повышения мыслительной деятельности человека, принять на себя ряд функций человека-эксперта или осуществлять деятельность в качестве ассистента лица, способного принимать решение в проблемной ситуации [7].

А основе анализа мирового опыта можно заключить, что технологию экспертных систем используют, чтобы решить различные типы задач, связанных с управлением, интерпретацией, диагностикой, планированием, конструированием, контролем и пр., в ряде проблемных областей, например, в нефтяной и газовой промышленности, энергетике, транспорте, медицине, космосе, металлургии, горном деле, химии, в области телекоммуникации и связи, экологии и пр.

В строительной отрасли технология экспертных систем также вызывает определённый интерес, это подтверждает ряд зарубежных и отечественных публикаций [13]. В нашей стране ряд исследований и разработок в области экспертных систем становятся частью государственных и отраслевых научно-технических программ.

В архитектуру экспертных систем входят различные модули, которые имеют различную реализацию, но их состав и взаимодействие имеют чёткое назначение. Создавая экспертную систему, разработчики направляют основные усилия не том, чтобы создать базу знаний, осуществить выбор моделей представления знаний и обозначить методы принятия решений [8]. Экспертные знания сочетают в себе теоретическое понимание проблемы и эмпирические правила (эвристики) для её решения. В экспертных системах реализуется мышление человека, а точнее - ряд элементов его мыслительной деятельности в процессе принятия решения, а также объем знаний, накопленных специалистами и описанных научной литературой и методическими и нормативными документами.

Чтобы осуществить автоматизированный поиск экспертного заключения о КТС строительного объекта при формализации декларативных знаний, используют технологию онтологического анализа [6], который призван исследовать и интерпретировать системные связи в сложных объектах, при меняя для этого методы и средства компьютерного моделирования. Термин «онтология» в теории искусственного интеллекта включает объем знаний, формально представленных в виде описания множества, включающего объекты, понятия и связи между ними [10].

Начало применения онтологического анализа выступает аналитическая работа, позволяющая выделить и объединить предметные знания, то есть сначала строится неформальная концептуальная модель знаний посредством определения набора основных понятий, а также отношений между ними. Чтобы оценить КТС конструкций, проводя обследование реальных строительных объектов, нужно учесть ряд всевозможных причин, 
которые приводят к изменению категории технического состояния. Сбор неформальной информации в базу знаний является стратегически важной и наиболее сложной задачей в разработке ЭС. Источниками знаний можно определить обширную базу практических и теоретических исследований в рамках технической диагностики конструкций зданий и сооружений [15], нормативную документацию, комплекс эвристических знаний и рассуждений специалистов.

По результатам исследования в рамках формирования онтологий различных типов строительных конструкций (балок, колонн, плит), для изготовления которых использован различный материал (древесина, железобетон, металл) производится декомпозиция множества контролируемых параметров технического состояния конструкций и связей между ними в виде иерархической четырёхуровневой структуры взаимосвязанных этапов решения.

Цель такого анализа - используя инструментальную оценку повреждённости (наличие и параметры дефектов, трещин), оценку состояния материалов, проведение ряда поверочных расчётов напряжённо-деформированного состояния элементов конструкций при учете имеющихся повреждений, проверку соответствия характеристик изучаемой конструкции и действующих нормативных требований касательно механической безопасности про извести определение категории технического состояния конструкции (является ли здание нормативным, работоспособным, ограниченно работоспособным, аварийным) [9].

Система автоматизированного обследования представлена следующими этапами работ:

- обмерными работами, проводимыми непосредственно на объекте;

• синхронизированной передачей данных эксперту;

- $\quad$ подготовкой данных в рамках выполнения прочностных расчётов и диагностики состояния конструкций;

• н непосредственным расчётом с использованием специализированных программ;

- $\quad$ оформлением ряда документов [11].

В процессе обмерных работ специалисты выполняют следующее:

- $\quad$ выбирают рабочую станцию. Рабочую станцию, представляющую собой моторизованный электронный тахеометр, устанавливают таким образом, чтобы была видимость всех опорных точек. После полученные координаты наблюдаемых точек вводят в систему управления электронного тахеометра. После все дальнейшие наблюдения осуществляют автоматически. Весь объем полученных измерений передается на NoteBook, архивируется и запоминается;

- $\quad$ выбираются опорные точки. Для съемки определяют ряд характерных точек каркаса здания (например, точки соединения элементов каркаса, точки обреза фундамента и т. д.);

- $\quad$ выбирают пульт оператора, расположенный на расстоянии от места обследования (Note Book c Bluetooth);

- $\quad$ проводят измерения приборами неразрушающего контроля и применяя экспрессдиагностику. В результате таких работ может быть оперативно получена информация, касающаяся различных параметров обследуемых конструкций. Будучи оборудованными Bluetooth устройством, они дают возможность оперативно передавать ряд измеренных величин эксперту в режиме синхронизации; 
- $\quad$ фотографируют имеющиеся дефекты фотоаппаратом, имеющим возможность подключения к $\mathrm{Wi}-\mathrm{Fi}$, что позволяет наглядно отобразить информацию об объекте и передать её в режиме On-line [14].

За счет оперативного получения данных эксперт может сразу сформировать дефектную ведомость.

Данные собираются непосредственно на объекте исследования и формируются посредством ноутбука, подключенного к Интернет. Получи необходимую информацию, главным специалистом (экспертом) формулируется задание помощникам в рамках продолжения обследования, причем больший акцент делается на детализацию информации о выявленных дефектах. Дефектную ведомость формирует главный эксперт в процессе проведения обследования, в диалоговом режиме сообщая помощнику такую информацию, как номер дефектов, их привязка к осям и листам подосновы планов и фасадов. Работу на объекте обследования сопровождают видеосъёмкой всех действий, а также аудиовизуальной информацией о фиксируемых дефектах.

За счет применения информационных технологий (IT) в процессе проведения «полевого» этапа работ появляется возможность получения и обработки информации в реальном режиме, повышения качества полученной информации и пр. [14].

Данный метод также позволяет использовать при дистанционном обследовании несущих строительных конструкций комбинированный метод, дающий возможность оценить прочностные характеристики и спрогнозировать целостность конструкций. Его можно применить как для существующих, так и для вновь создаваемых зданий и сооружений. Преимущества использования информационных технологий при обследовании зданий следующие:

- исполнитель работ в «полевых» условиях на объекте может отследить сбор данных;

- $\quad$ возрастает продуктивность работы за счёт того, что значительно сокращается время для сбора и последующей обработки информации;

- возрастает точность контроля ряда параметров (геометрических, конструктивных) и появляется возможность автоматизации процесса получения информации об объекте, а также расчета и оценки прочностных характеристик исследуемых строительных конструкций.

Таким образом, в качестве основного критерия принятия решений о необходимости проведения капитального ремонта или о признании здания аварийным выступает категория технического состояния здания. На принимаемые решения оказывает влияние объективность и достоверность информации, предоставляемой экспертами, но достаточно часто такая информация находится под влиянием их субъективного мнения. Применение информационных технологий позволяет учитывать разброс индивидуальных мнений. Данные технологии позволяют дать строгое математическое описание расплывчатых утверждений касательно технического состояния здания на текущий момент.

\section{ЛИТЕРАТУРА}

1. Асаул В.В., Александрова Е.Б., Кришталь В.В., Семенова С.В. Минимизация рисков формирования эффективных интеграционных образований в инвестиционно-строительной сфере. СПб: АНО «ИПЭВ», 2011. 364 с. 
2. Воронин И.А., Изатов В.А. О возможности определения сметной стоимости строительных объектов с использованием BIM проектирования // Материалы всероссийской конференции «Экономические и организационно-управленческие проблемы развития строительного комплекса России», 2014. С. 11.

3. Арбузова Е.К. Роль и значение информационных систем в строительстве // Экономическое развитие России: тенденции, перспективы. Материалы II Международной научно-практической конференции. Нижний Новгород. 2016. С. $5-9$.

4. Соколов, В.А. Определение категорий технического состояния строительных конструкций зданий и сооружений с использованием вероятностных методов распознавания // Предотвращение аварий зданий и сооружений: сб. науч. тр. Выпуск 9. - М., 2010. - С. 375-387.

5. Добромыслов, А.Н. Диагностика повреждений зданий и инженерных сооружений / А.Н. Добромыслов. - М.: МГСУ, 2006. - 256 с.

6. Гучкин, И.С. Диагностика повреждений и восстановление эксплуатационных качеств конструкций - М.: АCB, 2001. - 171 с.

7. Кашеварова, Г.Г. Интеллектуальная автоматизация инженерного обследования строительных объектов // International Journal for Computational Civil and Structural Engineering. - 2017. - T. 13. - № 3- C. 42-57.

8. Селютина Л.Г. Системный подход к решению задач в сфере проектирования и управления строительством // Kant. 2015. № 2 (15). С. 71-72.

9. Мамаев А.Е. Методика мониторинга календарного графика строительства на основе ВІМ технологии // Фундаментальные исследования. 2017. № 8-2. С. 270275.

10. Ильченко Д.П., Симанкина Т.Л., Романович М.А. Корректировка календарного плана ремонтно-строительных работ на основе метода замещения плановых работ // Вестник гражданских инженеров. 2013. № 2 (37). С. 125-130.

11. Павлов, А.С. Передача информации и распознавание объектов в системах строительного проектирования. Текст / А.С. Павлов. М.: Фонд «Новое тысячелетие», 2003. - 272 с.

12. Системы для надёжной, безопасной и эффективной эксплуатации зданий. URL: http://www.sodislab.com/rus/about/ (дата обращения 29.01.2020).

13. Мамаев А.Е., Шарманов В.В. Контроль инвестиционно-строительного проекта на основе ВІМ-модели здания // Актуальные проблемы гуманитарных и естественных наук. 2016. № 1-3. С. 83-86.

14. Придвижкин С.В., Баженов О.В., Шевелева A.E. Bim-Lean-синергия. Инструменты технологического обеспечения бережливого строительства // Экономика и управление: проблемы, решения. 2017. Т. 2, № 6. С. 98-104.

15. Селютина Л.Г. Методологические основы формирования и развития системы правления процессом преобразования жилого фонда крупного города // Общество. Среда. Развитие (Terra Humana). 2009. № 2. С. 212-218. 


\title{
Shesterikov Yuri Alekseevich \\ National research Moscow state university of civil engineering, Moscow, Russia Research and education center for testing structures E-mail: norb-89@mail.ru
}

Stifeeva Olga Alekseevna

National research Moscow state university of civil engineering, Moscow, Russia E-mail: norb-89@mail.ru

\section{Information systems and technologies for inspection of buildings and structures}

\begin{abstract}
The article describes the features of usingo information systems and technologies for inspection of buildings and structures. The author notes that the main criterion for making decisions about the need for major repairs or the recognition of the building as an emergency is the category of technical condition of the building. To assess the category of technical condition of structures, conducting a survey of real construction objects, you need to take into account a number of possible reasons that lead to a change in the category of technical condition. Collecting informal information into the knowledge base is a strategically important and most difficult task in the development of ES. Sources of knowledge can be defined as an extensive base of practical and theoretical research in the framework of technical diagnostics of buildings and structures, regulatory documentation, a set of heuristic knowledge and reasoning of specialists.

The ability to automate the solution of tasks of expert activity in the framework of diagnostics of building structures in conditions of uncertainty should be sought in the field of artificial intelligence systems. Decisions are influenced by the objectivity and reliability of information provided by experts, but often such information is influenced by their subjective opinion. The use of information technology allows you to take into account the diversity of individual opinions. Among the advantages of using information technologies for building surveys is that the contractor can track data collection in the "field" conditions on the site; the productivity of work increases due to the fact that the time for collecting and subsequent processing of information is significantly reduced; the accuracy of monitoring a number of parameters (geometric, structural) increases and it becomes possible to automate the process of obtaining information about the object, as well as calculating and evaluating the strength characteristics of the studied building structures.
\end{abstract}

These technologies allow us to give a strict mathematical description of vague statements about the technical condition of the building at the moment.

Keywords: building structures; expert systems; information technologies; inspection; parameters; emergency condition; major repairs; expert

\section{REFERENCES}

1. Asaul V.V., Aleksandrova E.B., Krishtal' V.V., Semenova S.V. (2011). Minimizatsiya riskov formirovaniya ehffektivnykh integratsionnykh obrazovaniy $\mathrm{v}$ investitsionnostroitel'noy sfere. [Risk minimization of the formation of effective integration entities in the investment and construction sector.] Saint Petersburg: Institute of problems of economic revival, p. 364.

2. Voronin I.A., Izatov V.A. (2014). O vozmozhnosti opredeleniya smetnoy stoimosti stroitel'nykh ob"ektov s ispol'zovaniem BIM proektirovaniya. [On the possibility of determining the estimated cost of construction projects using BIM design.] p. 11. 
3. Arbuzova E.K. (2016). Rol' i znachenie informatsionnykh sistem v stroitel'stve // Ehkonomicheskoe razvitie Rossii: tendentsii, perspektivy. [The role and importance of information systems in construction // Economic development of Russia: trends, prospects.] Nizhny Novgorod, pp. 5-9.

4. Sokolov V.A. (2010). Opredelenie kategoriy tekhnicheskogo sostoyaniya stroitel'nykh konstruktsiy zdaniy i sooruzheniy $\mathrm{s}$ ispol'zovaniem veroyatnostnykh metodov raspoznavaniya. [Definition of categories of technical condition of building structures of buildings and structures using probabilistic recognition methods.] Moscow, pp. 375387.

5. Dobromyslov A.N. (2006). Diagnostika povrezhdeniy zdaniy i inzhenernykh sooruzheniy. [Diagnostics of damage to buildings and engineering structures.] Moscow: Moscow State University of Civil Engineering, p. 256.

6. Guchkin I.S. (2001). Diagnostika povrezhdeniy i vosstanovlenie ehkspluatatsionnykh kachestv konstruktsiy. [Diagnostics of damages and restoration of operational qualities of structures.] Moscow: ASV, p. 171.

7. Kashevarova G.G. (2017). Intelligent automation of engineering survey of construction sites. International Journal for Computational Civil and Structural Engineering, 3(13), pp. 42-57 (in Russian).

8. Selyutina L.G. (2015). A systematic approach to solving problems in the field of design and construction management. Kant, 2(15), pp. 71-72 (in Russian).

9. Mamaev A.E. (2017). Methodology for monitoring the construction schedule on the basis of BIM technology. Basic Research, 8-2, pp. 270-275 (in Russian).

10. Il'chenko D.P., Simankina T.L., Romanovich M.A. (2013). Correction of the schedule of repair and construction works on the basis of the method of replacing planned works. Bulletin of Civil Engineers, 2(37), pp. 125-130 (in Russian).

11. Pavlov A.S. (2003). Peredacha informatsii i raspoznavanie ob"ektov v sistemakh stroitel'nogo proektirovaniya. [Information transfer and object recognition in building design systems.] Moscow: New Millennium Foundation, p. 272.

12. Sodislab. (n.d.). Systems for the reliable, safe and efficient operation of buildings. [online] Available at: http://www.sodislab.com/rus/about/ (in Russian). [Accessed 29.01.2020].

13. Mamaev A.E., Sharmanov V.V. (2016). Control of the investment and construction project based on the BIM model of the building. Actual problems of the humanities and natural sciences, 1-3, pp. 83-86 (in Russian).

14. Pridvizhkin S.V., Bazhenov O.V., Sheveleva A.E. (2017). Bim-Lean Synergy. Tools for technological support of lean construction. Economics and Management: Problems, Solutions, 6(2), pp. 98-104 (in Russian).

15. Selyutina L.G. (2009). Methodological foundations of the formation and development of a government system by the process of transforming the housing stock of a large city. Society. Wednesday. Development (Terra Humana), 2, pp. 212-218 (in Russian). 